livraisons

d'Histoire

de l'Architecture

\section{Livraisons de l'histoire de l'architecture}

42 | 2021

2001-2021 / numéro anniversaire

\title{
Des mots architecturés au temps mouvant
}

Architectural words in moving time

Von Architekturworten und den Zeitläuften

\section{Annette Viel}

\section{OpenEdition}

\section{Journals}

Édition électronique

URL : https://journals.openedition.org//ha/4077

DOI : 10.4000/lha.4077

ISSN : 1960-5994

Éditeur

Association Livraisons d'histoire de l'architecture - LHA

Référence électronique

Annette Viel, « Des mots architecturés au temps mouvant », Livraisons de l'histoire de l'architecture [En ligne], 42 | 2021, mis en ligne le 11 décembre 2021, consulté le 11 décembre 2021. URL : http:// journals.openedition.org/lha/4077 ; DOI : https://doi.org/10.4000/lha.4077

Ce document a été généré automatiquement le 11 décembre 2021.

Tous droits réservés à l'Association LHA 


\section{Des mots architecturés au temps mouvant}

Architectural words in moving time

Von Architekturworten und den Zeitläuften

\section{Annette Viel}

« La connaissance est une navigation dans un océan d'incertitudes en se ravitaillant dans des îles de certitudes ${ }^{1}$. » Edgar Morin

1 J'ai largué les amarres, quitté ma rive fluviale, senti le vent gonfler les voiles de ma modeste embarcation laquelle voguait allègrement dans le courant des flots d'une mer infinie. À vive allure, toutes voiles déployées, j'ai pris plaisir à naviguer vers cette prometteuse rade initiée par Jean-Michel Leniaud et Béatrice Bouvier en cette année symbolique que fut 2001. J'y ai alors trouvé un mouillage sécure qui abritait une riche flotte imprégnée de passionnants récits inspirés de l'histoire unique qu'est celle de l'architecture. Puis, sans crier gare et à mon grand étonnement, les mots architecturés au gré du temps mouvant ont vite rattrapé les miens et, presque tout naturellement, des passerelles insoupçonnées se sont façonnées. La structuration de mon propos découvrait, dans la rade réservée aux artisans de la revue, des ancrages signifiants. 


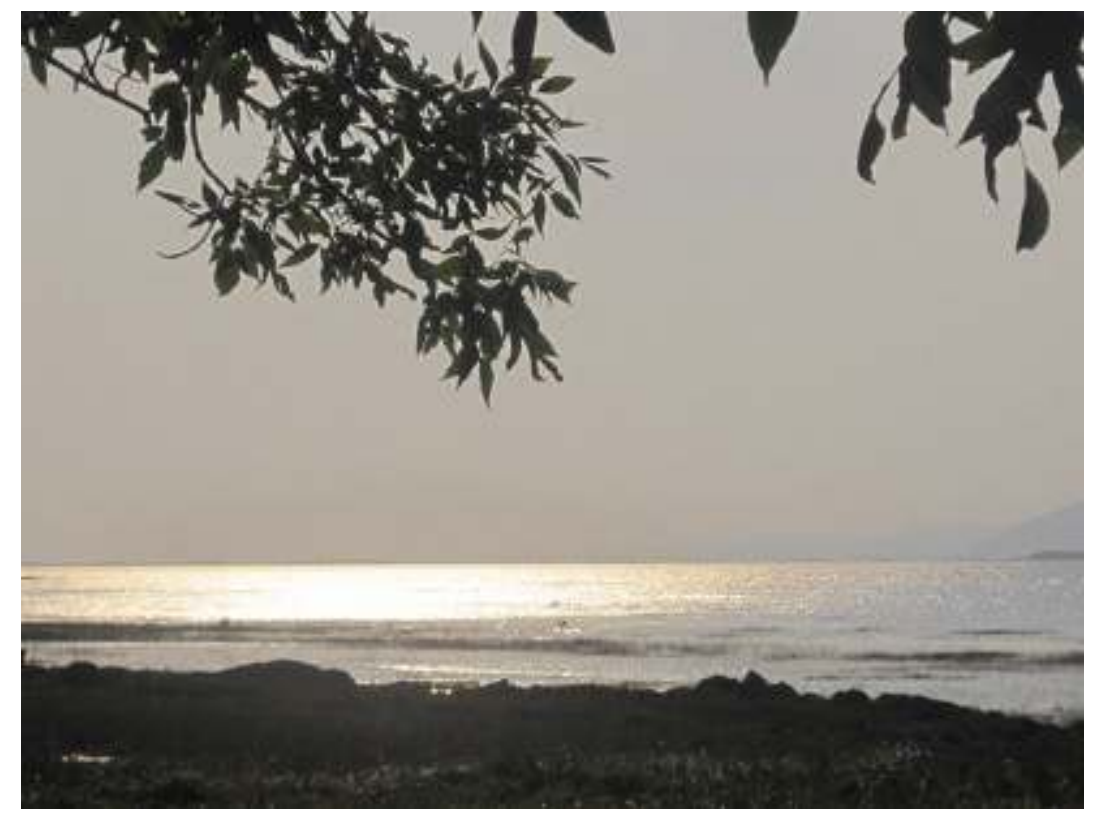

(c) Cliché de l'auteur

2 J'y ai ressenti ce privilège dont bénéficiaient de jeunes acteurs qui, au sein de la publication, trouvaient place pour partager leurs recherches et visions mettant en scène mille et une facettes de l'histoire de l'architecture. Des mots clés ont balisé mon sillage! Des mots images ont ponctué mes ancrages! Des mots évocateurs ont enrichi mon bagage! Des mots tels des amers glanés au fil des traces codifiées au sein des 20 ans de la Livraison d'histoire de l'architecture et des arts qui s'y rattachent.

\section{Architecture : la commande aux ouvriers...}

3 Par-delà sa racine étymologique d'origine grecque, l'architecture n'exprime-t-elle pas la capacité d'entrer en résonance avec la manière d'habiter l'espace dans une époque déterminée? Les mots de Marguerite Yourcenar trouvèrent résonance à mes réflexions: "Construire, c'est collaborer avec la terre: c'est mettre une marque humaine sur un paysage qui en sera modifié à jamais ${ }^{2}$.» Cette marque humaine nous invite à se prendre au jeu de l'incontournable quête de sens via les strates archéologiques d'une architecture inscrite au cœur des pays/paysages qui portent notre histoire, des pays/paysages en mouvance. Ce jeu stratigraphique qui sillonne les quelques 40 exemplaires produits durant les vingt ans de la revue, a vite imposé ses repères historiques tels quatre points cardinaux :

- Architectures des établissements d'enseignement supérieur $\left(\mathrm{n}^{\circ} 13\right)$ : des ancrages au cœur d'une urbanité en mouvement

- Émotions patrimoniales ( $\mathrm{n}^{\circ} 17$ \& 22) : dans le respect de l'esprit des lieux

- Histoire du (des?) patrimoine (s) ( $\left.\mathrm{n}^{\circ} 33\right)$ : strate, stratigraphie, stratégie

- Le Phare et l'architecte ( $\left.{ }^{\circ} 24\right)$ : balises inédites, éclairages innovants, amers prometteurs

Et, étonnamment, comme si un sextant s'était glissé dans ma main au beau milieu de la nuit claire, le quatrième repère : Le Phare et l'architecte, a étendu ses horizons vers une constellation d'étoiles. Les lumières se transformèrent alors en autant de phares qui, je 
l'espère, participeront dans un futur prochain, tout en solidarité, aux nombreuses recherches éclairant la route vers une planète plus consciente de ses gestes, traçant ainsi une voie ouverte en direction d'une paix davantage en dialogue avec ancrages et devenirs.

\title{
Architectures des établissements d'enseignement supérieur. Des ancrages au cœur d'une urbanité en mutation
}

\author{
«Bénéficiant également de son statut particulier \\ de société d'éducation non confessionnelle, \\ dégagée des pesanteurs de la tutelle ministérielle, \\ elle peut ainsi réaliser dans la pierre, très tôt \\ dans l'histoire des constructions scolaires \\ parisiennes, ses propres réponses architecturales \\ à la question de l'enseignement. » Annabelle \\ Lebarbé, « Le collège Sainte-Barbe de Paris : des \\ frères Labrouste à Ernest Lheureux ", Livraisons \\ d'histoire de l'architecture, no 13, 2007, p. 137-148.
}

5 Je me suis sentie interpellée par ces lieux d'enseignement dont les multiples strates racontent les valeurs de ceux qui les ont architecturés et habités, des lieux au cœur des cités en mouvement, des lieux osant édifier leurs «propres réponses architecturales à la question de l'enseignement. " Comme tous les projets auxquels j'ai contribué, lesquels trouvaient inévitablement écho au sein des communautés où ils prenaient place, il en va de même pour ces architectures d'établissements d'enseignement supérieur. Au cours du siècle dernier, le contexte de la globalisation planétaire a généré une approche transdisciplinaire créatrice de paramètres conceptuels novateurs notamment au regard du développement de projets qui se retrouvent maintenant fortement sous influence internationale. Nous sommes à vivre une période transitoire qui, par exemple, concernant les enseignements universitaires, s'est ouverte vers de nouvelles pistes d'éducation et de création tout en conservant les bases permanentes de la recherche incluant la notion de valeur.

6 Pour stimuler des visions architecturales en prise avec les grands enjeux paysagers contemporains, notamment en matière d'environnement, il importe d'éviter de retenir une approche méthodologique reposant sur deux positions extrêmes : «tradition » et «modernisation». Les nouvelles approches doivent privilégier un dialogue interculturel et interdisciplinaire favorisant la construction de connexions significatives entre la façon traditionnelle d'habiter les paysages et le désir d'y inscrire une modernité innovante dont l'architecture témoignera. Pour respecter la diversité qui y a pris forme au gré des époques, il faut connaître les cultures, les gens et les territoires d'intervention particulièrement à travers les multiples strates occupationnelles.

7 Trop souvent portés jusqu'alors par nos propres champs disciplinaires, il me semble toutefois que nous sommes de plus en plus à la recherche d'une matrice originale capable de moduler ces transversalités dont nous avons grand besoin stimulant ainsi des approches globales et globalisantes d'autant plus lorsqu'il s'agit de mettre en valeur 
le territoire. Des mots repères, reflets de l'inévitable mutation, ont émergé au cours de ma réflexion :

\section{2. Fleuve Saint-Laurent}

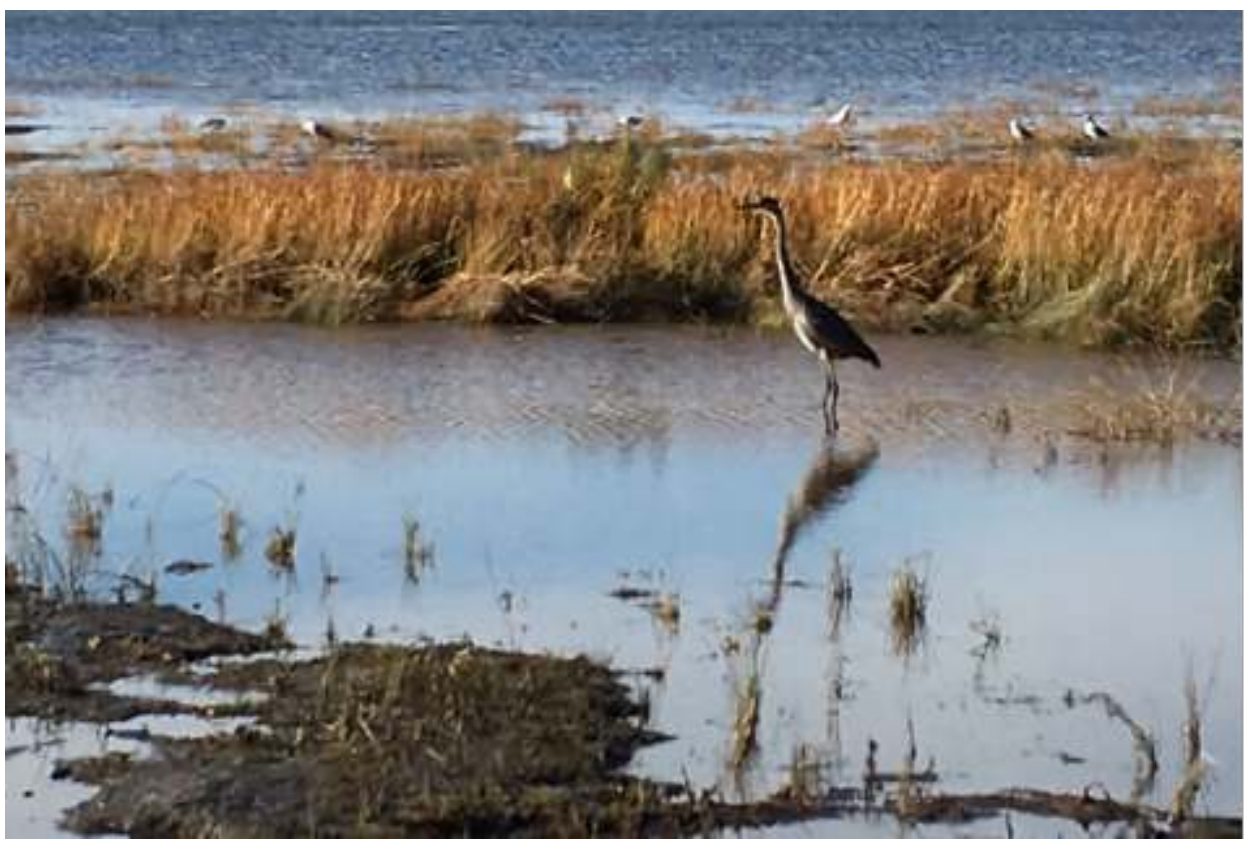

(c) Cliché de l'auteur

\section{$8 \quad$ Mixité}

- Des mondes et des univers

- Des traditions et des modernités

- De l'individuel et du collectif

- De l'ici et de l'ailleurs

- Du conscient et de l'inconscient

9 Migration

- Des cultures

- Des frontières nature/culture

- Des pratiques

- Des approches

- Des représentations

\section{Dynamisme}

- Au sein de pratiques respectueuses du principe voulant que les cultures soient nécessairement vivantes et enracinées dans des territoires en mutation dont les paysages portent des traces tangibles et intangibles. Dès lors, il importe de créer une synergie de projet humaniste en concertation avec le milieu et initiant un dialogue ouvert entre tradition et modernité.

\section{Connaissances}

- Plurielles dès l'amont notamment au regard de l'histoire, du territoire, de la culture, des enjeux, du contexte contemporain, etc. 
- Soutenues durant tout le projet stimulant ainsi les expériences pluridisciplinaires, au sein du milieu d'action, par le dialogue et le partage de façon à réfléchir et agir ensemble ; s'ouvrir à l'autre, transcender ses propres frontières.

- Pérennes en aval, assurant la continuité sur l'ensemble du territoire en créant une vision diachronique et synchronique inscrite dans une dynamique économique et sociale contemporaine.

Éthique professionnelle ouverte

- Afin que tout au cours du projet, le processus favorise des échanges à l'échelle locale, régionale, nationale et internationale tout en respectant la spécificité culturelle et évitant la perte de sens noyé dans les enjeux économiques et politiques. Les projets doivent être menés en concertation avec le milieu; l'apport venant de l'extérieur doit permettre la création d'une synergie fédératrice entre l'ici (le local) et l'ailleurs (le global) : le glocal.

\section{Émotions patrimoniales. Dans le respect de l'esprit des lieux}

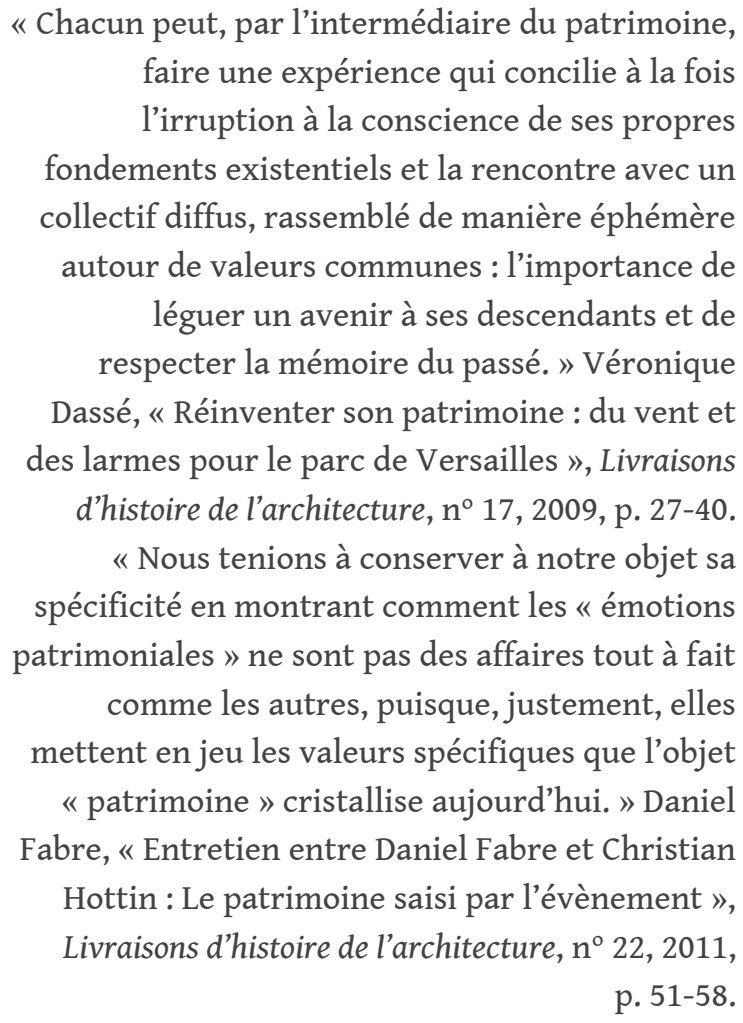

Dès mes débuts au sein de l'univers muséal et patrimonial, j'ai ressenti la nécessité d'aborder les lieux en transposant l'approche sémiologique découverte lors de mes études en histoire de l'art. Comme une œuvre d'art ne peut être saisi dans son essence profonde sans qu'elle ne soit d'abord ressentie, j'étais convaincue qu'il en allait de même pour les lieux patrimoniaux, qu'ils soient nature ou culture. Ayant grandi en pleine nature, aux abords du grandiose fleuve Saint-Laurent, je savais d'instinct combien il était important d'apprivoiser l'espace en le ressentant tout en développant des outils diversifiés pour mieux le connaître avant d'agir. Le lieu représente un espace pluriel, complexe et signifiant qui ne peut être appréhendé qu'en portant attention à l'ensemble des signes qui le constituent. Ainsi, au fil de mes investigations 
patrimoniales, la notion de respect de l'esprit des lieux a rapidement intégré l'approche conceptuelle privilégiée alliant la tradition ancestrale du territoire à celles de l'interprétation et de la recherche du sens dont sont porteurs ces lieux ${ }^{3}$.

\section{3. L'incontournable reliance Nature/Culture}

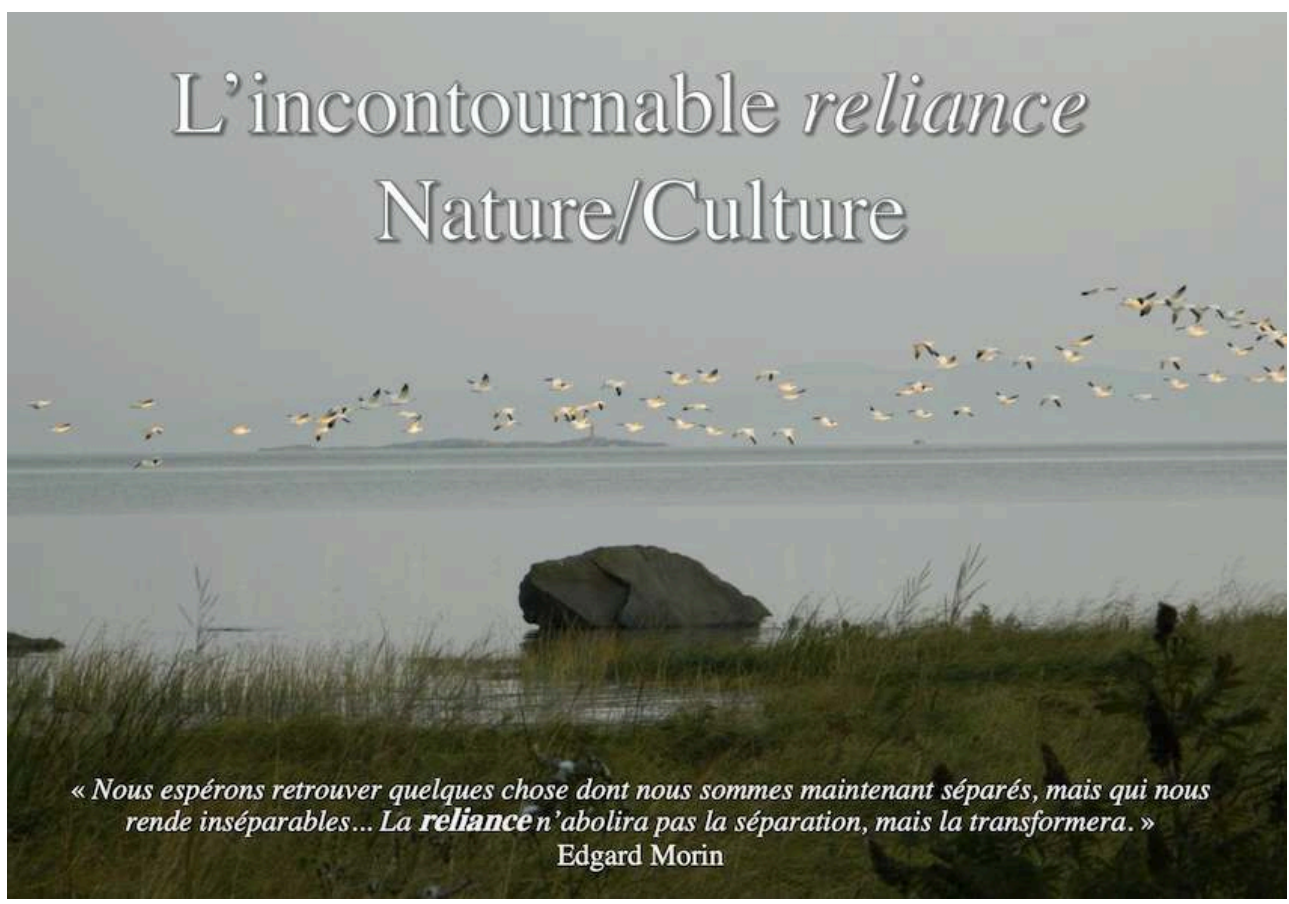

(c) Cliché de l'auteur

De manière fort judicieuse et très enrichissante pour les générations qui les ont suivis, plusieurs historiens du $\mathrm{XX}^{\mathrm{e}}$ siècle ont questionné le rapport au temps à la fois dans sa représentativité que dans les formes privilégiées pour l'étudier. Certains d'entre eux ont innové en initiant des approches inédites. Par exemple, ce fut le cas en France lorsqu'a été créée l'École des Annales portée dans un premier temps par les Braudel, Bloch, Febvre, puis les Duby, Leroy-Ladurie, Furet, Le Goff. Ces chercheurs ont rejeté la démarche historique traditionnelle davantage centrée sur le fait événementiel au profit de la longue durée tout en essayant de s'ouvrir aux autres sciences humaines et, dès lors, à la pluridisciplinarité, intégrant notamment les sphères de l'anthropologie, de la sociologie, de l'ethnologie et de la géographie. Aux débuts des années quatre-vingt, Pierre Nora poussa plus loin la réflexion en adjoignant la notion polysémique et polymorphe de « lieux de mémoire » concrétisant ainsi le passage de l'idée convergente de nation vers une prise en compte multiforme des territoires davantage fragmentés. Mémoire et histoire s'ouvraient l'une à l'autre initiant ainsi des manières contemporaines d'interpréter. La reconnaissance conceptuelle de l'idée d'une mémoire collective avait été étudiée au début du siècle dernier par d'autres chercheurs comme le sociologue Maurice Halbwachs qui, dans ses écrits publiés quelques années avant la création de l'École des Annales, approchait la notion mémorielle dont on parle tant aujourd'hui. 


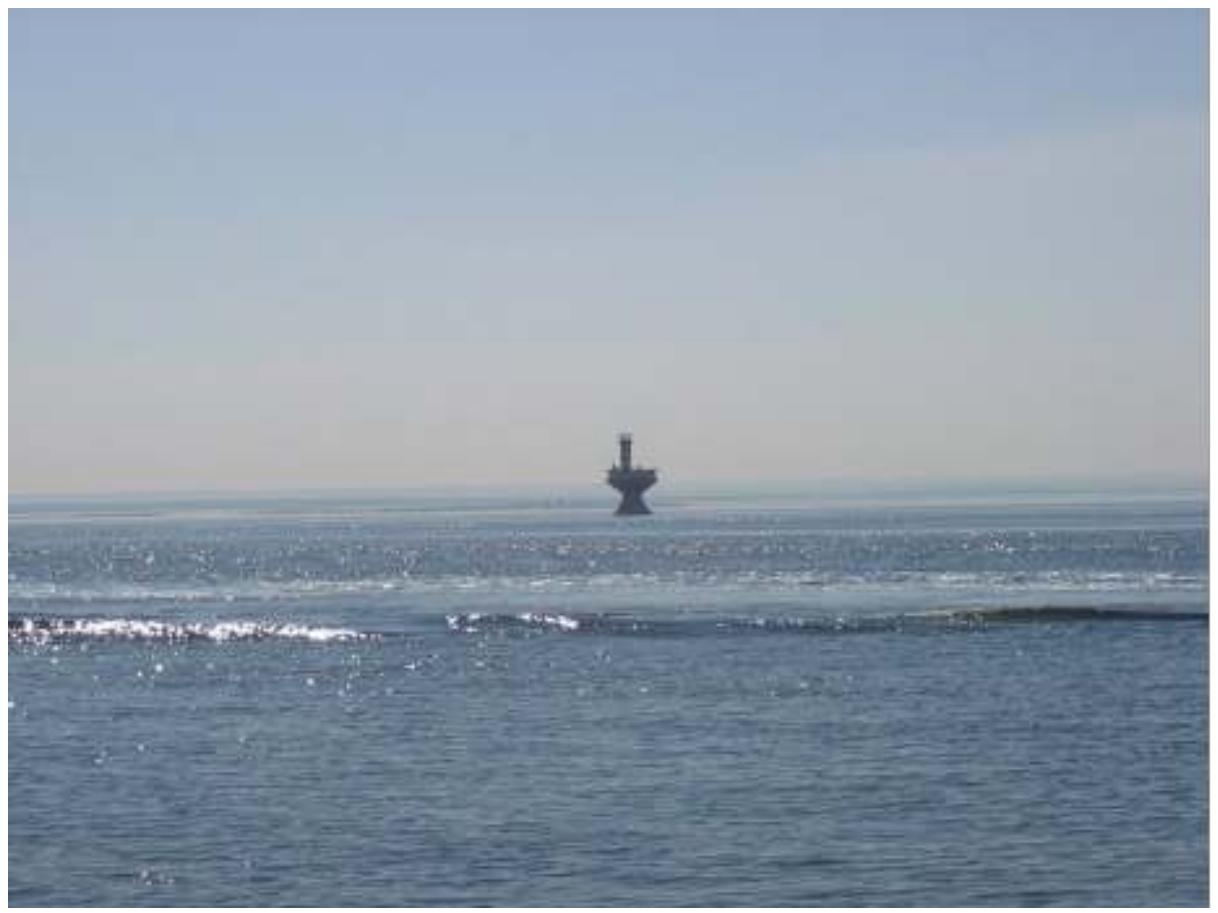

(c) Cliché de l'auteur

15 Paul Ricœur a, plus que tout autre, tenté d'approcher la mémoire différemment s'appuyant sur le fait d'une connaissance interprétative qui se laisse déchiffrer au même titre que les récits historiques et les discours. Ricœur admet l'importance des médiations linguistiques, narratives et surtout éthiques pour accéder à l'interprétation dont l'objet serait l'agir humain. Le philosophe oppose le JE, fondamentalement égocentrique, au SOI éminemment relationnel insistant sur l'importance du dialogue avec l'autre. Ricœur plaide "en une revendication de la mémoire contre l'histoire ${ }^{4}$." "Ce n'est donc pas avec la seule hypothèse de la polarité entre mémoire individuelle et mémoire collective qu'il faut entrer dans le champ de l'histoire, mais avec celle d'une triple attribution de la mémoire: à soi, aux proches, aux autres ${ }^{5}$. " Les lieux patrimoniaux constituent des espaces de représentation du passé et, du même coup, d'appréhension de l'avenir. Miroirs d'une époque, ils témoignent de ses valeurs, représentant autant de passerelles entre JE, SOI et L'AUTRE, autant d'interprétations qui révèlent, traduisent et donnent à voir des facettes des sociétés auxquelles ils sont redevables. Et les ancrages architecturaux n'échappent pas aux interprétations d'autant qu'ils constituent des témoins phares de bien des lieux patrimoniaux. Comme le souligne Daniel Fabre cité plus haut: "les "émotions patrimoniales » ne sont pas des affaires tout à fait comme les autres, puisque, justement, elles mettent en jeu les valeurs spécifiques que l'objet « patrimoine » cristallise aujourd'hui. »

\section{Histoire du(des?) patrimoine(s). Strate, stratigraphie, stratégie}

« Restituer la généalogie des projets permet de dépersonnaliser le débat public contemporain et 
de comparer dans la durée les options et les blocages de l'aménagement. » Loïc Vadelorge, " Diagnostic patrimonial et histoire urbaine", Livraisons d'histoire de l'architecture, $\mathrm{n}^{\circ} 33,2017$, p. 87-94.

Lorsqu'on développe un projet architectural, il importe de prendre en compte la globalité du paysage où il s'inscrit. Cette approche permet d'enrichir son développement d'une vision stratigraphique intégrée et ainsi, de baliser un cadre d'action à la fois efficace et respectueux des valeurs patrimoniales qui en signent l'ensemble. Dès lors une nouvelle stratégie de projet se met en place intégrant une dimension patrimoniale vivante qui stimule un dialogue signifiant entre les modernités : celles du passé comme celles d'un présent respectueux des traces de son histoire tout en s'ouvrant vers demain.

\section{5. Fleuve Saint-Laurent}

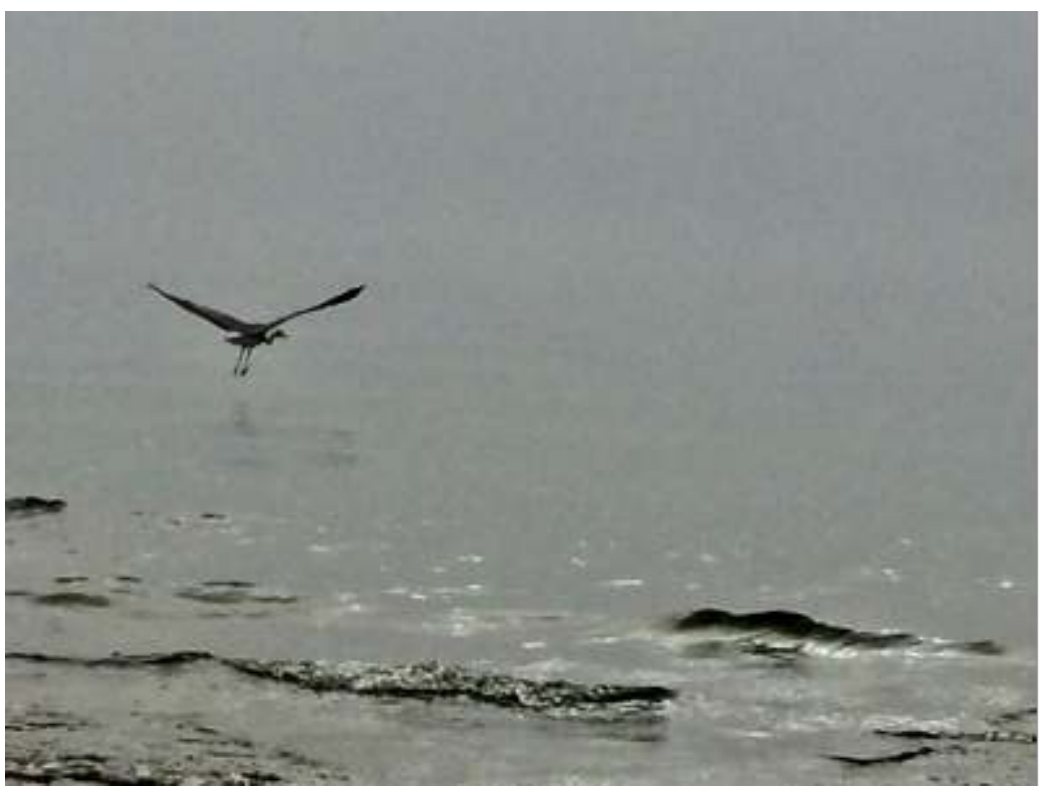

(C) Cliché de l'auteur

17 L'homme contemporain habite maintenant la planète terre, initiant des manifestations culturelles davantage internationalisées. La notion d'identité, telle que ressentie en ce début $\mathrm{XXI}^{\mathrm{e}}$ siècle, opère l'entrée en scène de nouvelles frontières créatrices de liens entre :

- Les temps historiques et contemporains

- Les espaces de proximité et du lointain

- Les nouvelles tribus internationales telles que sont les chercheurs, les architectes, les artistes, les artisans, les musiciens, les gens d'affaires, les universitaires, etc.

Des tribunes d'échanges novatrices se redéfinissent sous l'enseigne du préfixe : trans dont le sens premier signifie à travers, au-delà :

- Transdisciplinarité

- Transfrontaliarité

- Transgénérationnalité

- Transversalité 
- Transculturalité

- Transcommunalité

- Transfonctionnalité

\section{Une expérience transdisciplinaire en trois temps: sens, science, conscience}

\section{6. Mont Saint-Michel}

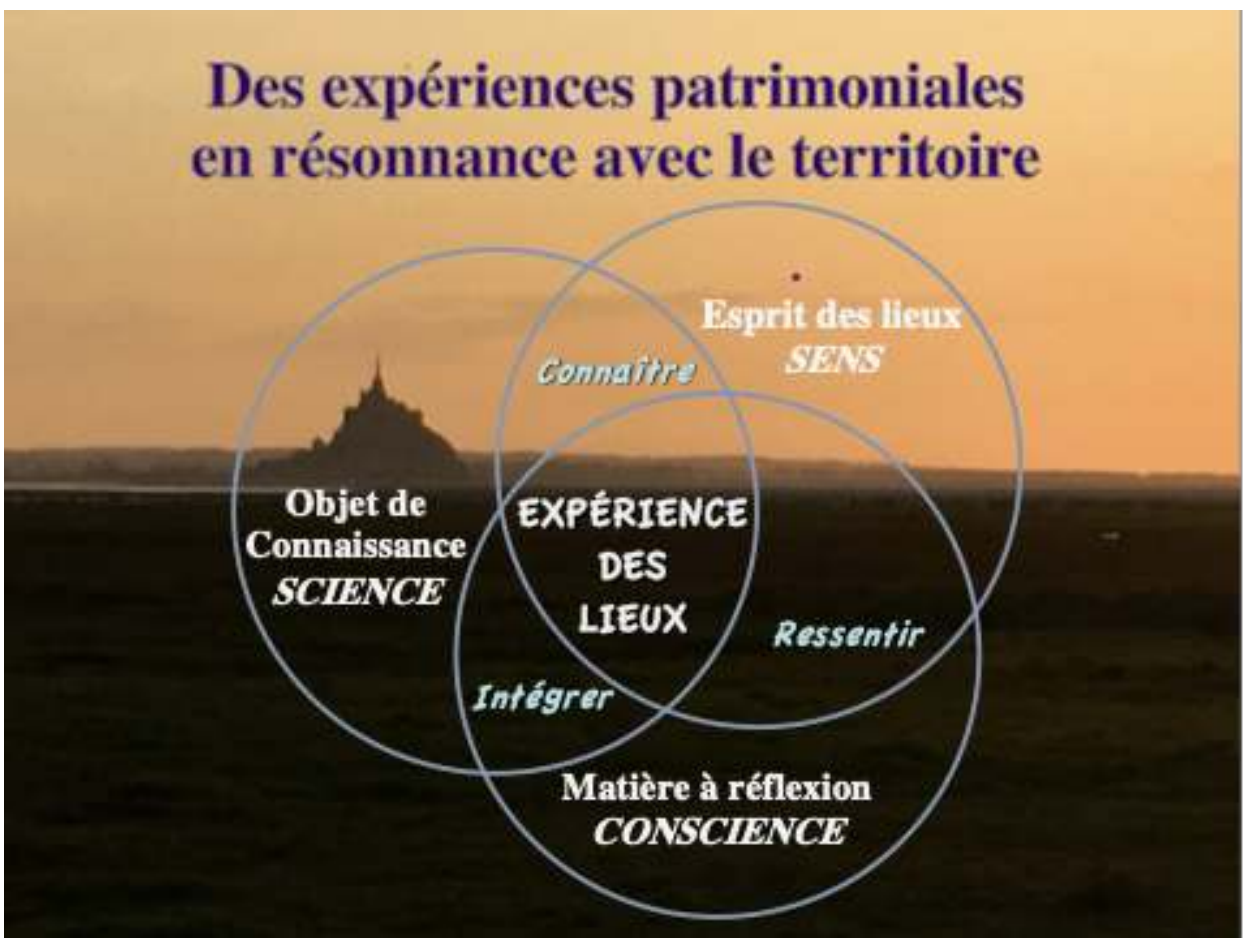

(c) Cliché de l'auteur

Fortement engagée au sein de la mise en valeur du patrimoine, dès le début des années quatre-vingt, j'ai initié une approche conceptuelle favorisant une alliance entre sens, science et conscience ; une approche porteuse des valeurs passées, présentes et à venir tout en suscitant la création d'une synergie conviant les différentes disciplines à partager leurs savoirs et leurs compétences; une approche à l'enseigne de la transdisciplinarité. Au premier principe du respect de l'esprit du lieu, se sont vite imposés deux autres éléments permettant de structurer une approche conceptuelle globale : la définition de l'objet de connaissance (le fait scientifique) et le développement d'une matière à réflexion. Ces trois axiomes allaient servir de base à l'énoncé des expériences patrimoniales offertes aux différents publics que ce soit dans un lieu nature ou culture. Les trois composantes de l'expérience: sens, science et conscience s'énoncent ainsi :

- L'expérience de sens révélée par l'émotion ressentie : l'esprit du lieu. Le lieu s'inscrit dans un territoire qui possède déjà une histoire et qui se vit au jour le jour. Le public perçoit l'ensemble de ce lieu : son paysage, son bâti, ses vestiges et les communautés qui l'habitent. Ce lieu reflète des valeurs et invite au dialogue autour d'une histoire nature/culture qui a laissé des traces reconnues tout comme celles qui, aujourd'hui, s'y manifestent. 
- L'expérience de connaissance (science) apportée par l'objet/sujet conservé, interprété et mis en valeur sur le site. La connaissance est transmise par le paysage, les objets collectionnés et les thématiques interprétées. Le public enrichit son univers de connaissance et de savoir grâce à ce qu'il découvre. Il intègre ces savoirs à sa propre expérience.

- L'expérience de conscience induite par la réflexion qui émerge de l'esprit du lieu ainsi que des savoirs interprétés et muséographiés. Le public intègre l'expérience vécue à son bagage personnel prolongeant bien au-delà du site ses découvertes, son ressenti et ce que lui a apporté, comme individu et comme citoyen, ce passage au lieu.

\section{Le phare et l'architecte. Balises inédites, éclairages innovants, amers prometteurs}

«Érigés en pleine nature, les phares se trouvent entièrement exposés à ses forces mais aussi, et surtout, au spectacle qu'elle offre, c'est-à-dire au perpétuel dynamisme du monde animé et inanimé qui se répète et se renouvelle sans cesse.

En tant que monuments, ils sont à même de s'insérer dans ce contexte comme s'ils en faisaient naturellement partie. Ils deviennent ainsi des témoins privilégiés du cycle vital de l'univers, ou plutôt, ils favorisent ainsi la prise de conscience de ce cycle, aussi bien chez ses utilisateurs que chez de simples observateurs. " Marcelo Puppi, « L'imagination des phares chez

Léonce Reynaud ", Livraisons d'histoire de l'architecture, $\mathrm{n}^{\circ} 24,2012$, p. 63-84.

Des paysages/phares porteurs de sens à partager 


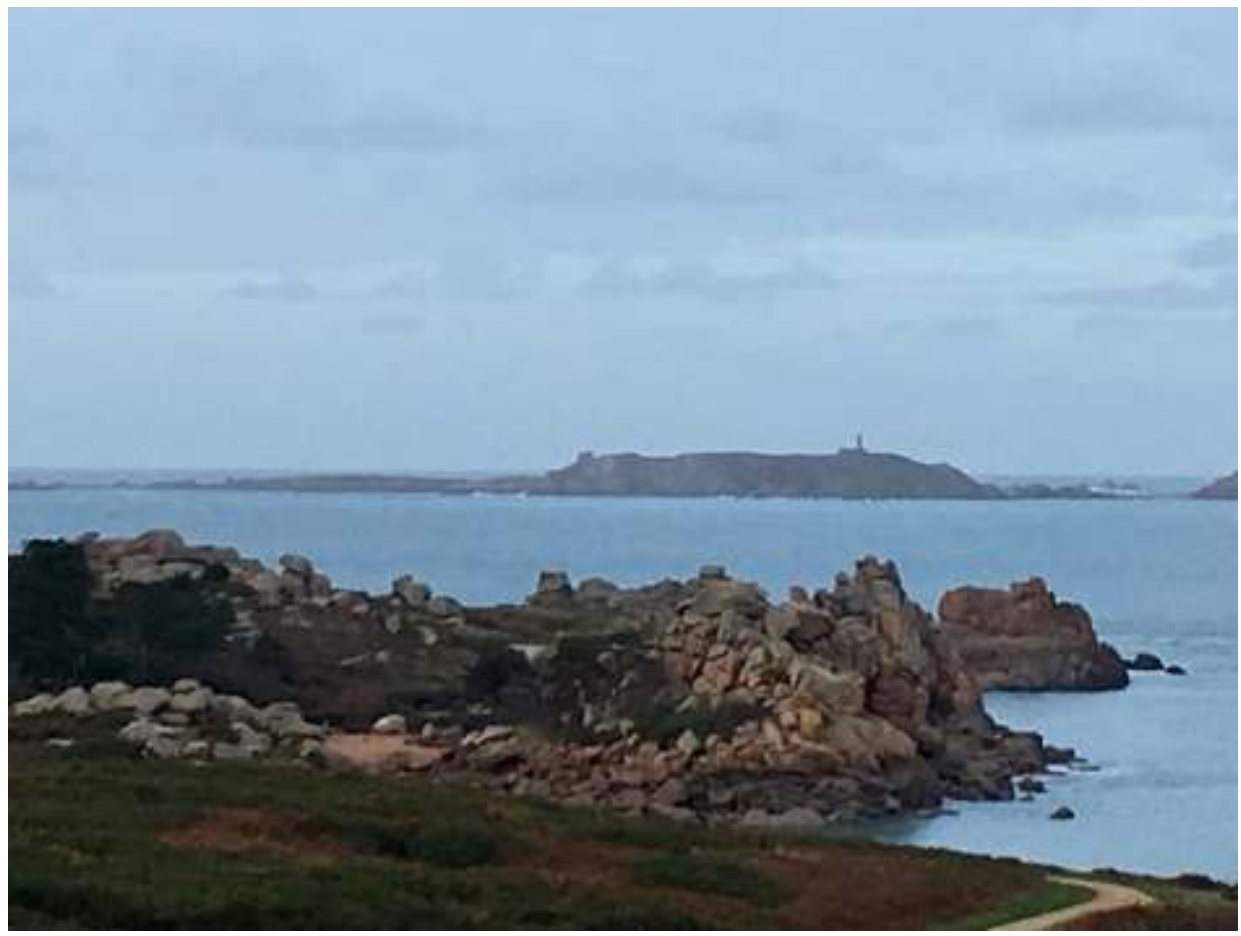

(c) Cliché de l'auteur

20 Les paysages sont comme des phares, témoins éclaireurs d'une époque baignée d'une quête de sens dans un monde en mutation. La connaissance de leur évolution favorise une approche intégrée encore plus lorsqu'il s'agit d'y développer de nouveaux projets qui, nécessairement, les modifieront. Plus que de simples représentations de la nature, les paysages font corps avec elle et, même si trop souvent et encore plus en ces temps de développement tous azimuts, on perçoit une certaine dichotomie entre paysage nature et paysage culture, les paysages n'en signent pas moins nos appartenances.

Car les paysages nous racontent. Ils portent les traces culturelles et symboliques de nos ancrages. Ils constituent des signes et des repères de nos modernités. Ils reflètent des choix de sociétés et traduisent les valeurs d'une époque. Une approche novatrice doit initier des outils favorisant une capacité à mettre en perspective nos paysages habités par une connaissance élargie et pluridisciplinaire incluant l'archéologie et l'histoire ${ }^{6}$.

\section{Des architectures/phares repères des mémoires en mouvement}

22 L'architecture constitue autant de phares symboles de nos manières d'habiter nos pays/paysages qui, aujourd'hui, interpellent les valeurs de ceux et celles qui les créent. Et comme l'a si clairement exprimé l'architecte italien Alberto Magnaghi : «Sous les coulées de lave de l'urbanisation contemporaine, survit un patrimoine territorial d'une extrême richesse, prêt à une nouvelle fécondation, par de nouveaux acteurs sociaux, capables d'en prendre soin et en voie d'émergence, surtout là où l'écart entre qualité de vie et croissance économique est le plus flagrant ${ }^{7}$. »

De nouveaux phares nature/culture affichant des éclairages innovants, proposant des amers prometteurs doivent baliser les projets architecturaux d'aujourd'hui, des architectures intégrées à la vie territoriale et ancrées dans une dynamique enracinée 
dans un développement dit durable. Des phares porteurs d'un éclairage signifiant dont l'architecture témoigne parvenant, entre autres, à tirer profit au maximum des nouvelles technologies sans en faire les frais et surtout en respectant l'esprit des lieux où ils inscrivent leur présent.

\section{Des phares miroirs d'une attitude humaniste entre soi et l'autre}

De plus en plus, il devient important d'orchestrer une synergie collective auprès de tous les acteurs impliqués au sein de la vie territoriale, plus particulièrement dans les domaines architecturaux, paysagers, urbanistiques, patrimoniaux, artistiques, géographiques, archéologiques, etc. On ne peut nier le rôle important qu'ils ont à jouer en tant que de passeurs de sens entre les modernités. D'une certaine manière, ces acteurs deviennent des médiateurs, des transmetteurs, des tisseurs de liens, des motivateurs permettant de relier les disciplines au service d'un milieu en relation signifiante avec les territoires où ils interviennent. Des territoires tissés d'histoires passées, d'histoires présentes qui sont à s'écrire, d'histoires qui éclairent l'avenir tels des phares polysémiques permettant aux navigateurs d'aujourd'hui de ne pas s'échouer sur des rivages d'où il sera difficile d'émerger.

Tisseur de nouvelles alliances entre tradition et modernité, entre tradition et innovation, entre tradition et contemporanéité, il faut assurer un ancrage inédit avec le milieu, un ancrage qui permet la mouvance, qui la guide, qui aide, qui dirige, qui balise la stimulante aventure architecturale d'aujourd'hui tout en gardant une porte ouverte vers une aventure dans des domaines inédits. Et comme Don Quichotte, ne perdons pas de vue nos rêves! Osons donner sens à ces architectures que nous imaginons pour mieux habiter ces pays/paysages qui nous habitent...

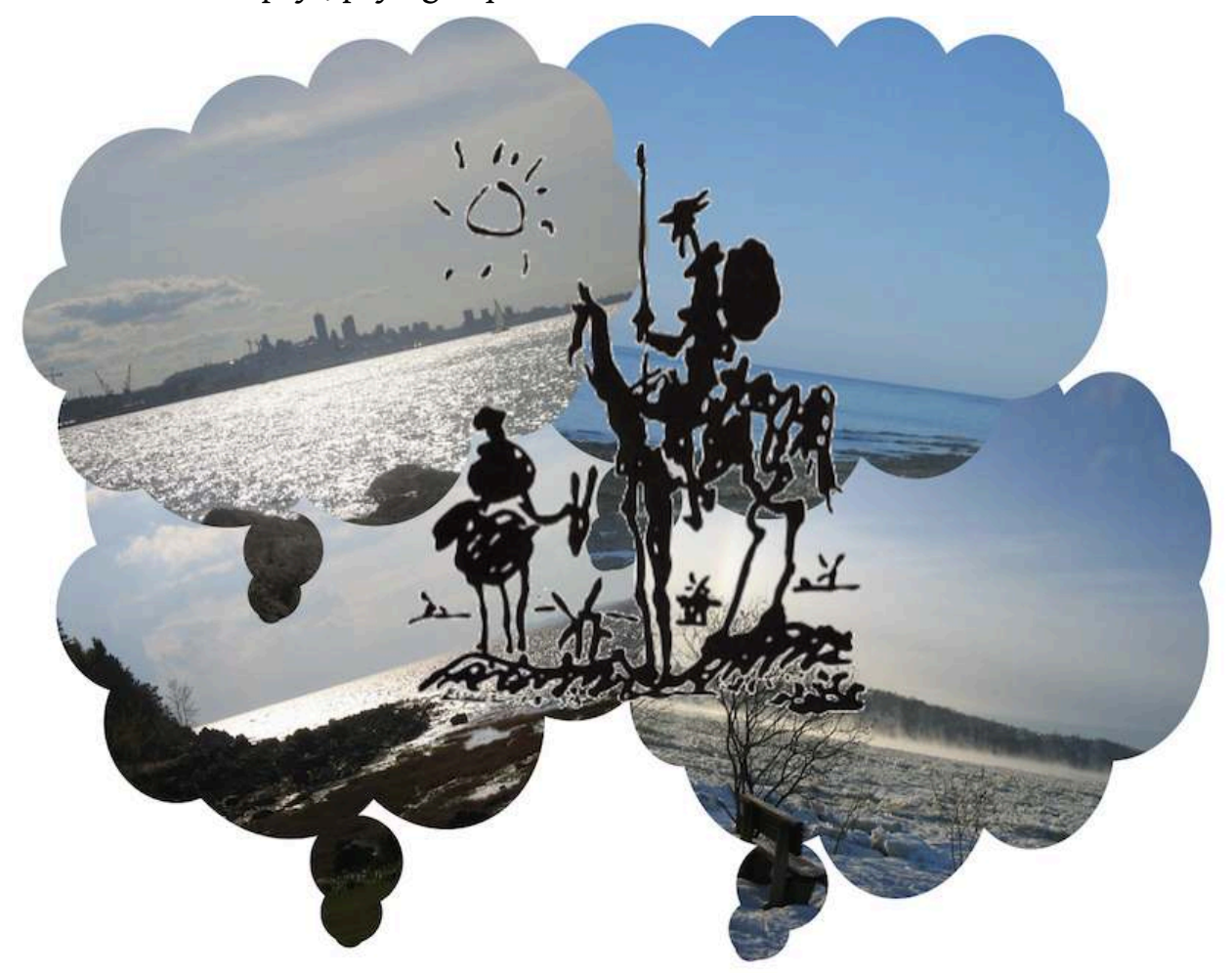

(c) Cliché de l'auteur 
«L'architecture, le plus incompris et le plus oublié des arts d'aujourd'hui, en est peut-être aussi le plus mystérieux et le plus nourri

d'idées. » Guy de Maupassant, La vie errante, chap. 1 « Lassitude », 1890, Éditions Paul

Ollendorff, p. 12.

\section{NOTES}

1. Edgar Morin, Leçons d'un siècle de vie, Paris, Denoël, 2021, p. 143.

2. Marguerite Yourcenar, Mémoire d'Hadrien, Paris, Folio ${ }^{\circ}$ 921, 1977, p. 140.

3. Annette Viel, Quand souffle l'esprit des lieux, ICOMOS, Colloque Où se cache l'esprit des lieux, Québec. https//www.icomos.org/quebec2008/cd/toindex/78_pdf/78-B3X3-152.pdf, 2008.

4. Paul Ricœur, La mémoire, l'histoire, l'oubli, Paris, Le Seuil, 2003, p. 106.

5. Paul Ricœur, La mémoire, l'histoire, l'oubli, op. cit., p. 163.

6. Annette Viel, «Créativité et territoire(s) en mouvement : créer pour mieux vivre et habiter la pluralité territoriale», Revue Ethnologies, Volume 38, $\mathrm{n}^{\circ}$ 1-2, Québec, Université Laval, 2016, p. 213-236.

7. Françoise Choay, Le patrimoine en questions. Anthologie pour un combat, La couleur des idées, Paris, Le Seuil, 2009, p. 209-210.

\section{RÉSUMÉS}

Quatre thèmes/numéros ont entrainé l'auteur vers une réflexion portée par des mots qui, tout naturellement, sont entrés en résonnance avec ses ancrages professionnels. Des mots qui sillonnent une mer riche d'aventures et d'ouvertures vers des contrées baignées de ce sens qui n'a de cesse de nourrir l'esprit des lieux au sein desquels, depuis la nuit des temps, l'architecture constitue une incontournable assise de l'histoire humaine.

Les thèmes abordés sont :

- Architectures des établissements d'enseignement supérieur $\left(n^{\circ} 13\right)$ : des ancrages au cœur d'une urbanité en mouvement.

- Émotions patrimoniales ( $\mathrm{n}^{\circ} 17$ \& 22) : dans le respect de l'esprit des lieux.

- Histoire du (des?) patrimoine (s) ( $\left.\mathrm{n}^{\circ} 33\right)$ : strate, stratigraphie, stratégie.

- Le Phare et l'architecte $\left(\mathrm{n}^{\circ} 24\right)$ : balises inédites, éclairages innovants, amers prometteurs.

Four topics/issues led the author to a reflection carried by words which, quite naturally, resonated with her professional anchors. Words that crisscross a sea full of adventures and openings towards regions bathed in this meaning that has never ceased to nourish the sense of 
place in which, since the dawn of time, architecture has been a must foundation of human history.

The topics covered are:

-Architectures of higher education institutions ( $\left.\mathrm{n}^{\circ} 13\right)$ : anchorages in the heart of urbanity in motion. -Heritage emotions ( $\left.\mathrm{n}^{\circ} 17 \& 22\right)$ : respecting the sense of place.

-Heritage(s?) history ( $\left.\mathrm{n}^{\circ} 33\right)$ : layer, stratigraphy, strategy.

-The lighthouse and the architect $\left(\mathrm{n}^{\circ} 24\right)$ : original signposts, innovative lightings, promising beacons.

Vier Ausgaben der „Livraisons d'histoire de l'architecture“ haben die Autorin zu Überlegungen zu einigen Begriffen angeregt, die ganz naturgemäß ihre eigenen beruflichen Tätigkeiten widerspiegeln. Es sind Worte, die einen ganzen Kosmos von Geschehnissen und Wegen des menschlichen Strebens hervorrufen, das den Geist jener Orte prägt, an welchen die Architektur seit Urzeiten eine unabdingbare Grundlage der Menschheitsgeschichte bildet.

Die behandelten Themen sind folgende:

-Architectures des établissements d'enseignement supérieur ( $\left.\mathrm{n}^{\circ} 13\right)$ : des ancrages au cœur d'une urbanité en mouvement

-Émotions patrimoniales ( $\left.\mathrm{n}^{\circ} 17 \& 22\right)$ : dans le respect de l'esprit des lieux

-Histoire du(des?) patrimoine (s) (n³3): strate, stratigraphie, stratégie

-Le Phare et l'architecte $\left(\mathrm{n}^{\circ} 24\right)$ : balises inédites, éclairages innovants, amers prometteurs

\section{INDEX}

Mots-clés : esprit des lieux, territoire, architecture, phare, patrimoine, mémoire

\section{AUTEUR}

\section{ANNETTE VIEL}

Annette Viel, muséologue, consultante internationale, a développé une approche originale de mise en valeur patrimoniale y intégrant la notion de respect de l'esprit des lieux. Elle a été professeur (2002-2007) au Muséum National d'Histoire Naturelle de Paris et à l'Université de Bourgogne (1999-2002). Elle a dirigé et collaboré à de multiples projets en muséologie et en patrimoine dans différents pays et continents. Elle prône une approche transdisciplinaire et diversifiée n'hésitant pas à prendre des voies inédites alliant arts et sciences. Elle a publié divers articles qui témoignent d'une nécessité d'œuvrer pour des lieux ouverts sur demain, des lieux incitant les publics à vivre une expérience de sens. En 2012, elle recevait le prix carrière remis par la Société des Musées Québécois (SMQ), précédé en 2008 du prix rayonnement international donné conjointement par l'Association des Musées Canadiens (AMC) et ICOM Canada. En 2018, elle fut nommée Chevalière des Palmes académiques françaises. Elle est membre du Comité scientifique international de la revue Culture \& Musées. 